\title{
LINEAR GROUPS AS STABILIZERS OF SETS
}

\author{
I. M. ISAACS ${ }^{1}$
}

\begin{abstract}
A finite linear group over an infinite field is necessarily the full setwise stabilizer of some finite subset of the corresponding vector space. If the group is absolutely irreducible, the set can be taken to be a single orbit.
\end{abstract}

Let $V$ be a finite dimensional vector space over a field $F$. For subsets $X \subseteq V$, let $\Gamma(X)$ denote the full setwise stabilizer of $X$ in the general linear group $\mathrm{GL}(V)$. If $G \subseteq \mathrm{GL}(V)$ is a subgroup, we consider the question of finding $X$ with $G=\Gamma(X)$.

If $F$ is finite or $G$ is infinite, such $X$ do not necessarily exist. Counterexamples of both types are easily found (for instance $G=\operatorname{SL}(V)$ ). We assume now that $G$ is finite and $F$ is infinite. Then $G=\Gamma(X)$ for some finite $X \subseteq V$. In fact we have more.

TheOREM 1. Let $G \subseteq \mathrm{GL}(V)$ be absolutely irreducible. Then $G=\Gamma(X)$ for some orbit $X$ of $G$ on $V$.

Theorem 2. Let $G \subseteq \mathrm{GL}(V)$ and let $Y \subseteq V$ be finite and $G$-invariant and span $V$. Then there exists an orbit $X$ of $G$ on $V$ such that $G=\Gamma(X \cup Y)$.

The key to the proofs of both theorems is the following fact which we shall not prove here. (This is Problem 21 on p. 177 of [1]. It follows from Lemma 1.2 of [2], which is in fact much stronger.)

LEMMA 3. Let $V$ be a vector space over an infinite field. Then $V$ is not the union of finitely many proper subspaces.

To prove Theorem 1 we need the following.

LEMMA 4. Let $G \subseteq \mathrm{GL}(V)$ be absolutely irreducible and let $m$ be a positive integer. Then there exist only finitely many subgroups $\Gamma \subseteq \mathrm{GL}(V)$ such that $\Gamma \supseteq G$ and $|\Gamma| \leqslant m$.

Proof. Since there are only finitely many isomorphism classes of groups of order $\leqslant m$, we may assume that there are infinitely many isomorphic $\Gamma \subseteq \mathrm{GL}(V)$ with $\Gamma \supseteq G$. Let $\Gamma_{0}$ be one of these. Since there are only finitely many isomorphisms of $G$ into $\Gamma_{0}$, we may assume that there exist infinitely

Received by the editors June 15, 1976.

AMS (MOS) subject classifications (1970). Primary $20 \mathrm{C} 99$.

Key words and phrases. Linear group, stabilizer of subset of vector space.

!:Research supported by N.S.F. grant MCS74-06398 A02. 
many $\Gamma \subseteq \mathrm{GL}(V)$ with isomorphisms $\theta: \Gamma \rightarrow \Gamma_{0}$ such that the restrictions of the $\theta$ to $G$ are all identical.

For each such $\Gamma$ and $\theta$, the map $\theta^{-1}: \Gamma_{0} \rightarrow \Gamma \subseteq \mathrm{GL}(V)$ is a representation of $\Gamma_{0}$ of degree equal to $\operatorname{dim} V$. Since the number of similarity classes of such representations is finite, we may assume that all of these $\theta^{-1}$ are similar representations.

We now have distinct $\Gamma_{1}, \Gamma_{2} \subseteq \mathrm{GL}(V)$ with isomorphisms $\theta_{i}: \Gamma_{i} \rightarrow \Gamma_{0}$ for $i \in\{1,2\}$ such that $G \subseteq \Gamma_{i}$ and the restrictions $\theta_{1}\left|G=\theta_{2}\right| G$. Also, there exists $P \in \mathrm{GL}(V)$ with $P^{-1} \theta_{1}^{-1}(x) P=\theta_{2}^{-1}(x)$ for all $x \in \Gamma_{0}$.

In particular, letting $x$ run over $\theta_{1}(G)=\theta_{2}(G)$ we conclude that $P$ commutes with all elements of $G$ and, hence, $P$ is a scalar matrix since $G$ is absolutely irreducible. Now $\theta_{2}^{-1}(x)=P^{-1} \theta_{1}^{-1}(x) P=\theta_{1}^{-1}(x)$ for all $x \in \Gamma_{0}$ and so $\Gamma_{2}=\Gamma_{1}$. This is a contradiction.

Proof of Theorem 1. If $X \subseteq V-\{0\}$ is any $G$-orbit, then $X$ spans $V$ since $G$ is irreducible. It follows that $\Gamma(X)$ permutes $X$ faithfully and so $|\Gamma(X)| \leqslant|X|$ !. Since $X$ is a $G$-orbit, we have $|X| \leqslant|G|$ and it follows from Lemma 4 that only finitely many groups $\Gamma(X)$ arise.

For each nonidentity element $t$ of each of the groups $\Gamma(X)$, let $W_{t}$ be the space of vectors fixed by $t$. Then $W_{t}$ is a proper subspace of $V$ and since there are only finitely many $t$, Lemma 3 applies and we can find some $v \in V$ which is not fixed by any nonidentity element of $\Gamma(X)$ for any $G$-orbit $X$.

Now let $X=v G$. By the choice of $v$ we have $|v \Gamma(X)|=|\Gamma(X)|$ and since $v \Gamma(X) \subseteq X$ and $|X| \leqslant|G|$ we have $\Gamma(X)=G$.

Proof of Theorem 2. Since $Y$ spans $V$, choose a basis $b \subseteq Y$ for $V$. Now use Lemma 3 to find $v \in V$ such that $v$ is not in any of the spaces spanned by proper subsets of $\mathfrak{b}$ and $v$ is not fixed by any nonidentity element of $\Gamma(Y)$. (Note that this can be done since $\Gamma(Y)$ is faithful on $Y$ and $Y$ is finite and hence, $\Gamma(Y)$ is finite.) Let $X=v G$. We shall show that $G=\Gamma(Y \cup \lambda X)$ for some scalar $\lambda \neq 0$.

Let $\Delta \subseteq \mathrm{GL}(V)$ be the setwise stabilizer of the set of one-dimensional subspaces spanned by the elements of $X \cup Y$. If $k$ is an element of the kernel of the action of $\Delta$ on this set then $k$ maps each $y \in \mathfrak{b}$ to a scalar multiple $\lambda_{y} y$. Also $v=\sum \alpha_{y} y$ with all $\alpha_{y} \neq 0$ and $k$ maps $v$ to $\lambda v$. This yields $\lambda v=v k=$ $\sum \alpha_{y} \lambda_{y} y$ and, hence, $\alpha_{y} \lambda_{y}=\alpha_{y} \lambda$ for all $y \in \mathfrak{b}$. It follows that all $\lambda_{y}=\lambda$ and $k$ is scalar multiplication by $\lambda$.

Let $Z \subseteq \mathrm{GL}(V)$ be the group of scalar multiplications on $V$. Then $Z \cong$ $F^{\times}$and $\Delta / Z$ permutes $\{F u \mid u \in X \cup Y\}$ faithfully. Thus $\Delta / Z$ is finite.

Write $\Gamma_{\lambda}=\Gamma(Y \cup \lambda X)$ and note that $\Gamma_{\lambda} \subseteq \Delta$ and $\left|\Gamma_{\lambda}\right|$ divides $m=$ $|X \cup Y|$ !. We claim that there are only finitely many different groups $\Gamma_{\lambda}$. It suffices to show that each of the finitely many cosets of $Z$ in $\Delta$ contains only finitely many elements of order dividing $m$. However, if $a \in \Delta$ and $b \in Z a$ with $a^{m}=1=b^{m}$, then $b=z a$ and $z^{m}=1$ with $z \in Z$. Since $Z \cong F^{\times}$and the equation $x^{m}=1$ has only finitely many solutions in $F$, the assertion follows. 
We can choose an infinite set $\Lambda \subseteq F^{\times}$such that all $\Gamma_{\lambda}$ are equal for $\lambda \in \Lambda$. Write $\Gamma=\Gamma_{\lambda}$ for $\lambda \in \Lambda$ and note that $\Gamma$ stabilizes $\bigcap_{\lambda \in \Lambda}(Y \cup \lambda X)$. The intersection is just $Y$ since no vector lies in infinitely many $\lambda X$. Thus $\Gamma \subseteq \Gamma(Y)$ and by the choice of $v$, we have $|v \Gamma|=|\Gamma|$. Take $\lambda \in \Lambda$ with $\lambda v \notin Y$. Then $Y \cap \lambda X=\varnothing$ and $\Gamma$ stabilizes $\lambda X$. Thus, $|G| \geqslant|X| \geqslant|v \Gamma|=$ $|\Gamma|$. Since $G \subseteq \Gamma$ we have $\Gamma(Y \cup \lambda X)=\Gamma=G$.

We close with the remark that if $G \subseteq \mathrm{GL}(V)$ is irreducible, it follows from Theorem 2 that $G=\Gamma(X)$ for some set $X$ which is a union of two $G$-orbits. However, unless $G$ is absolutely irreducible, there does not necessarily exist a single orbit which suffices. For example, let $G$ be cyclic of order $n>2$ and act faithfully on real 2-space by rotation. If $X$ is any orbit of nonzero vectors, then $\Gamma(X)$ is a dihedral group of order $2 n$.

\section{REFERENCES}

1. I. N. Herstein, Topics in algebra, Xerox, Lexington, 1975. MR 50 \#9456.

2. D. S. Passman, Infinite group rings, Dekker, New York, 1971.

Department of Mathematics, University of Wisconsin, Madison, Wisconsin 53706 